\title{
Percutaneous access to coronary arteries in patients after transcatheter aortic valve implantation procedures - is it a real problem?
}

\author{
Piotr Chodór ${ }^{1}$, Krzysztof Wilczek², Roman Przybylski³ ${ }^{3}$ Jerzy Nożyński ${ }^{4}$, Łukasz Włoch ${ }^{1}$, Zbigniew Kalarus ${ }^{1}$ \\ ${ }^{1}$ Department of Cardiology, Congenital Heart Diseases and Electrotherapy, Medical University of Silesia, Silesian Center for Heart Diseases, \\ Zabrze, Poland \\ ${ }^{2} 3^{\text {rd }}$ Department of Cardiology, Medical University of Silesia, Silesian Center for Heart Diseases, Zabrze, Poland \\ ${ }^{3}$ Department of Cardiac Surgery and Transplantology, Medical University of Silesia, Silesian Center for Heart Diseases, Zabrze, Poland \\ ${ }^{4}$ Department of Histopathology, Silesian Center for Heart Diseases, Zabrze, Poland
}

Adv Interv Cardiol 2019; 15, 3 (57): 274-282

DOI: https://doi.org/10.5114/aic.2019.87880

\begin{abstract}
A bstract
Transcatheter aortic valve implantation (TAVI) is still developing and changing our approach to treating patients with severe symptomatic aortic stenosis. Aortic stenosis frequently coexists with coronary artery disease. Both diseases have similar risk factors for their development and one should expect a future progression of coronary artery disease. The current guidelines have expanded TAVI indications to include intermediate-risk patients, and perhaps they will be expanded to include low-risk patients in the future. Survival after TAVI in younger patients will depend on the durability of the aortic valves and methods of coronary artery disease treatment. This paper presents some aspects of performing coronary angiography and percutaneous coronary intervention in patients who had TAVI performed using the two most popular aortic valves - balloon expandable aortic valves (Edward Sapien/ Edward Sapien XT/Sapien 3) and self-expandable aortic valves (CoreValve/Evolut R) - on the basis of several examples. This paper also focuses on technical aspects associated with a proper implantation of aortic valves to ensure easy access to coronary arteries, as well as on possible problems when the implantation is not optimal. We discuss interactions between the structure of the aortic valve stent, catheters, commissures of new aortic valves, and coronary ostia.
\end{abstract}

Key words: transcatheter aortic valve implantation, coronary percutaneous intervention, coronary artery disease.

\section{Introduction}

Aortic stenosis frequently coexists with coronary artery disease. Transcatheter aortic valve implantation (TAVI) procedures are being performed more and more often in patients with medium and low risk of surgical treatment, and the patients are younger and younger. In older patients, coronary heart disease has an ambiguous effect on the life span in short-term observations [1]. According to the UK Registry, coronary heart disease may affect the life span not earlier than after 5 years after the TAVI procedure [2]. Life expectancy of younger patients after TAVI will depend on the durability of the valve and the access to coronary arteries. There are reports on coronary angiographies and $\mathrm{PCl}$ procedures in patients after TAVI [3-5].

Theoretically some valves (Edwards Sapien, Sapien 3, Jena Valve, Accurate, Lotus) provide relatively easy access to the coronary arteries, while some of them make the access more difficult, depending on the density of the mesh or the presence of a new commissure at the level of the coronary ostia.

Some examples of access to coronary arteries after TAVI procedures used in our material are presented below. In the future, both selection of the valves and/or the method of valve implantation should be provided to ensure free access to the coronary arteries as well as effective percutaneous treatment of coronary heart disease in patients after TAVI.

\section{Examples}

\section{Example I}

A 76-year-old patient before a TAVI procedure underwent percutaneous coronary intervention $(\mathrm{PCI})$ of the left

\section{Corresponding author:}

Piotr Chodór MD, Department of Cardiology, Congenital Heart Diseases and Electrotherapy, Medical University of Silesia, Silesian Center for Heart Diseases, 2 Szpitalna St, 41-800 Zabrze, Poland, e-mail: chodor_piotr@go2.pl

Received: 25.02.2019, accepted: 18.04.2019. 
main coronary artery (LM) with stent implantation to the $L M$ and left anterior descending (LAD) artery, and a stent to the ostium of the circumflex artery (CX). Thirty-two months after the implantation of the Edwards Sapien XT 26 aortic valve, the patient was admitted to our hospital for acute coronary syndrome (ACS).

Access to the coronary arteries was easily obtained. The guiding catheter engaged the orifice of the left coronary artery (LCA) above the stent structure of the prosthesis. A critical ostial in-stent restenosis in the $C x$ was observed (Figure $1 \mathrm{~A}$, red arrow) and plain old balloon angioplasty (POBA) with a final kissing balloon (Fig. 1 B, red arrow) was performed using a drug-eluting balloon (DEB) with a good angiographic result and significant clinical improvement (Figures 1 C, D, red arrow).

\section{Example II}

Figures 2 A-E show cases of high implantation of Edwards Sapien XT and Sapien 3. High valve implantation may cause the stent structure to cover the ostium of the left coronary artery and the sinotubular junction (STJ) (Figures $2 \mathrm{~A}-\mathrm{E}$, red arrows). It is likely to result in impeded or almost impossible access to the coronary arteries.
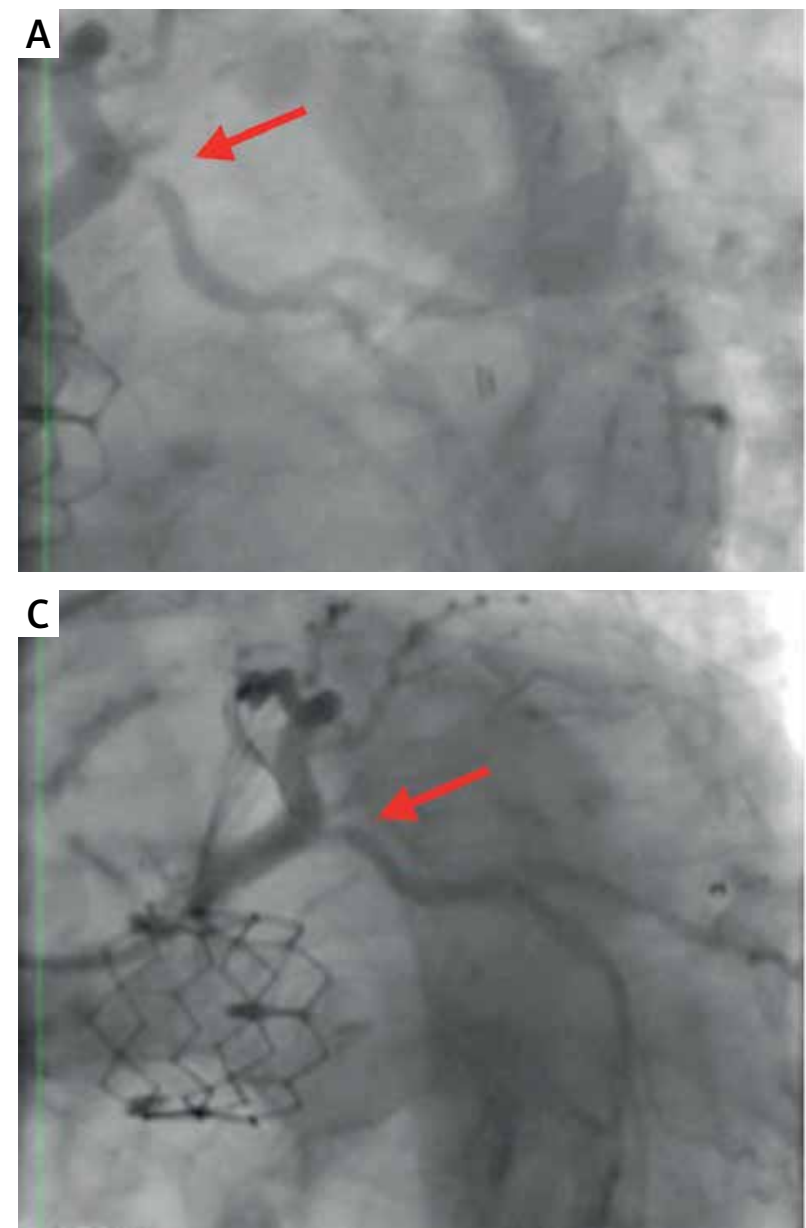

In other cases, when the aortic bulb is narrow or shallow, the valve may block access to the coronary ostia even after optimal implantation.

The position of new commissures opposite the ostia of the coronary arteries could be an additional impediment. If the ostia of the coronary arteries are located low, and the implantation is performed high, it may cause them to be located below the level of the outer sealing skirt (Figure 2 B).

\section{Example III}

Coronary angiography in a 79-year-old patient after TAVI with the CoreValve 29. The patient was admitted with the symptoms of ACS. Coronary angiography was performed through the right radial artery. A right coronary catheter (Judkins 3,5-6 F) passed between the valve stent and aortic bulb to enter an atypical origin of the right coronary artery (Figure $3 \mathrm{~A}$, red arrow). A Whisper Extra Support guidewire was used to stabilize the catheter and to provide proper injection to the right coronary artery (RCA) (Figure 3 A). Similarly, two Whisper Extra Support guidewires were used to intubate the left coronary artery with a left coronary Amplatz 16 Fr catheter
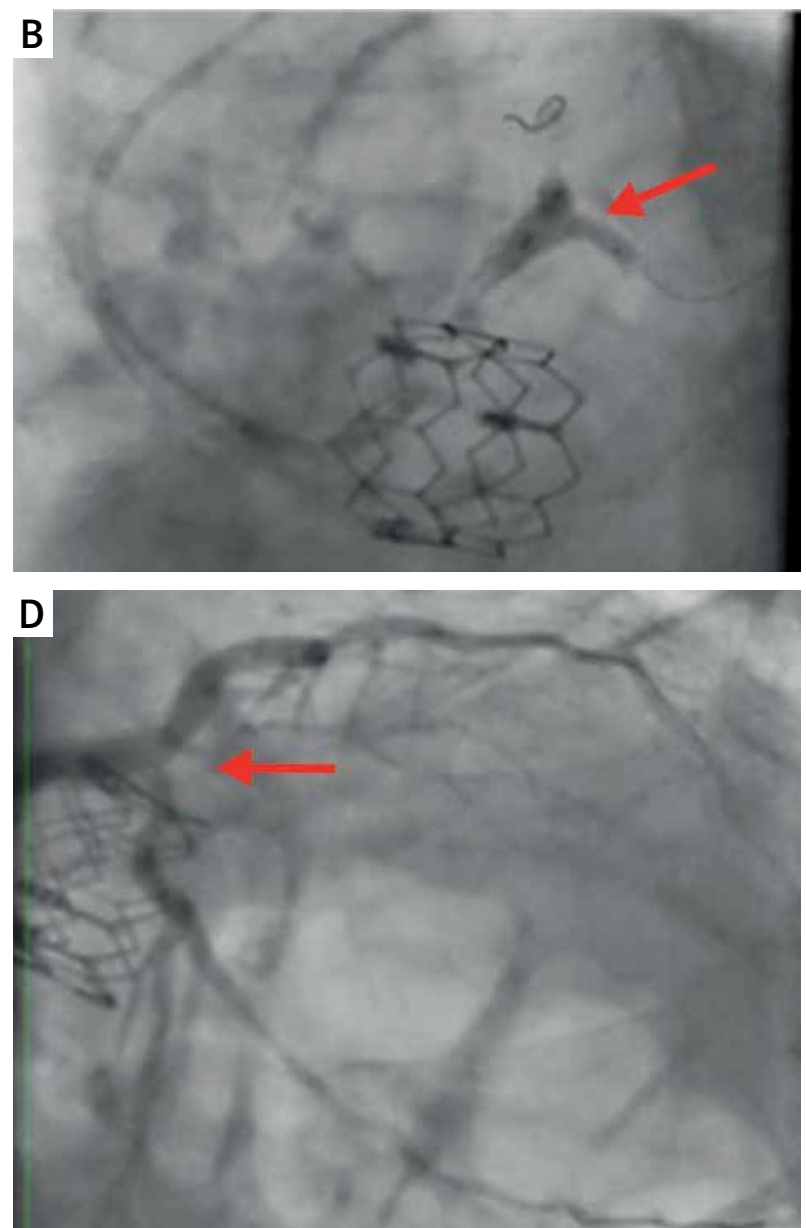

Figure 1. Easy access to the coronary arteries after implantation of Edward Sapien XT 26 valve 

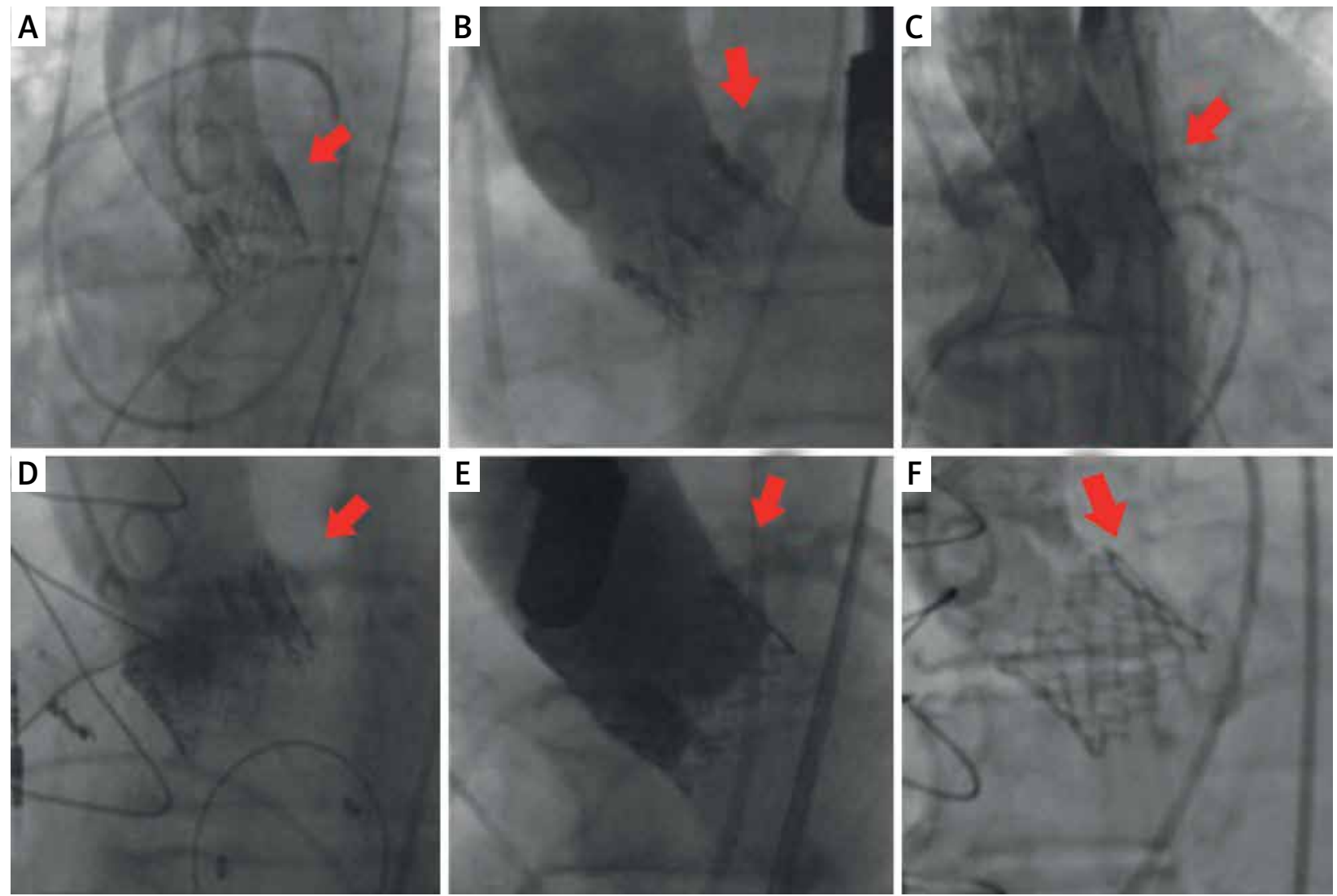

Figure 2. Possible difficulties in access to the coronary arteries after Edward Sapien XT and the Sapien 3 valve implantation
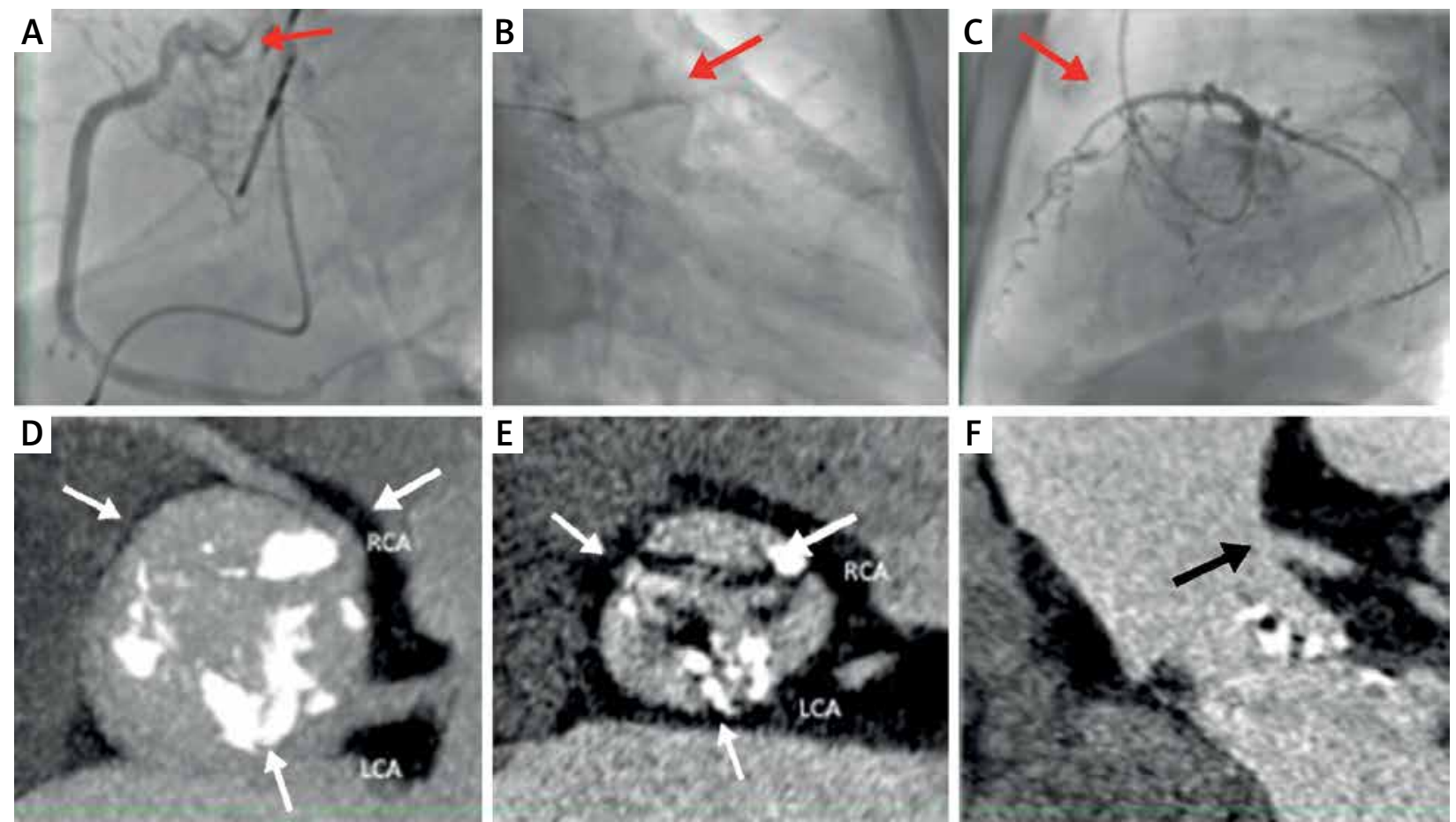

Figure 3. Difficulties in performing coronary angiography after implantation of the CoreValve 29 prosthesis with difficult anatomy of the coronary arteries 
and to stabilize it (Figures 3 B, C, red arrows). In this case, the catheter passes through the struts of the aortic valve stent. Coronary angiography did not show any significant changes in the coronary arteries.

A multi-slice computed tomography (MSCT) examination performed before TAVI showed an unusual origin of the right coronary artery from the left coronary sinus (Figures $3 \mathrm{D}$, E, white arrows - the commissure of the native valve). Both ostia originated high from the sinus (Figures $3 \mathrm{D}, \mathrm{F}$, black arrow - the origin of the LCA).

\section{Example IV}

An 84-year-old patient after TAVI with the CoreValve 26, with renal insufficiency on dialysis, was admitted due to suspected stenosis of the implanted valve. Due to reported chest pain, a coronary angiography was performed. Because of the difficulties with intubation of the left coronary artery with Judkins Left 4 and Amplatz Left 1 catheters, contrast medium was injected non-selectively (Figures 4 A, B, red arrows). The next figure shows the position of the coronary catheter inserted inside the valve stent; however, it is located far from the left coronary artery orifice (Figure $4 \mathrm{~B}$ ). Intubation of the right coronary artery orifice failed. The right coronary artery was non-selectively visualized by contrast injection to the aortic bulb through the pig-tail catheter (Figure $4 \mathrm{C}$ red arrow).

MSCT shows a dense mesh of the CoreValve stent and it was observed that the struts were located at the level of the right and left coronary artery ostia (Figures $4 \mathrm{D}, \mathrm{E})$. Furthermore, we can observe difficult conditions for the intubation of the right coronary artery in the presence of the valve struts and the unfavorable origin of the right coronary artery upwards from the aortic bulb (Figure $4 \mathrm{D}$ ). The upper part of the valve stent closely adhered to the aortic wall, which made it impossible to perform coronary angiography by passing the catheters between the aortic wall and the stent struts (Figure $4 \mathrm{D}$ ). The commissure of a new valve is located opposite the right and left coronary artery and along the stent struts. It was impossible to selectively intubate the right and left coronary artery (Figure $4 \mathrm{E}$, red arrows).

\section{Example V}

2.5 months after the TAVI procedure with an Evolut $R 26$ prosthesis, an 84-year-old patient was admitted to our hospital with the symptoms of ACS after fainting. Before the TAVI procedure, the patient underwent $P C I$ with drug-eluting stent (DES) implantation to the LM-LAD and obtuse marginal branch (OM) 1. The patient had multiple
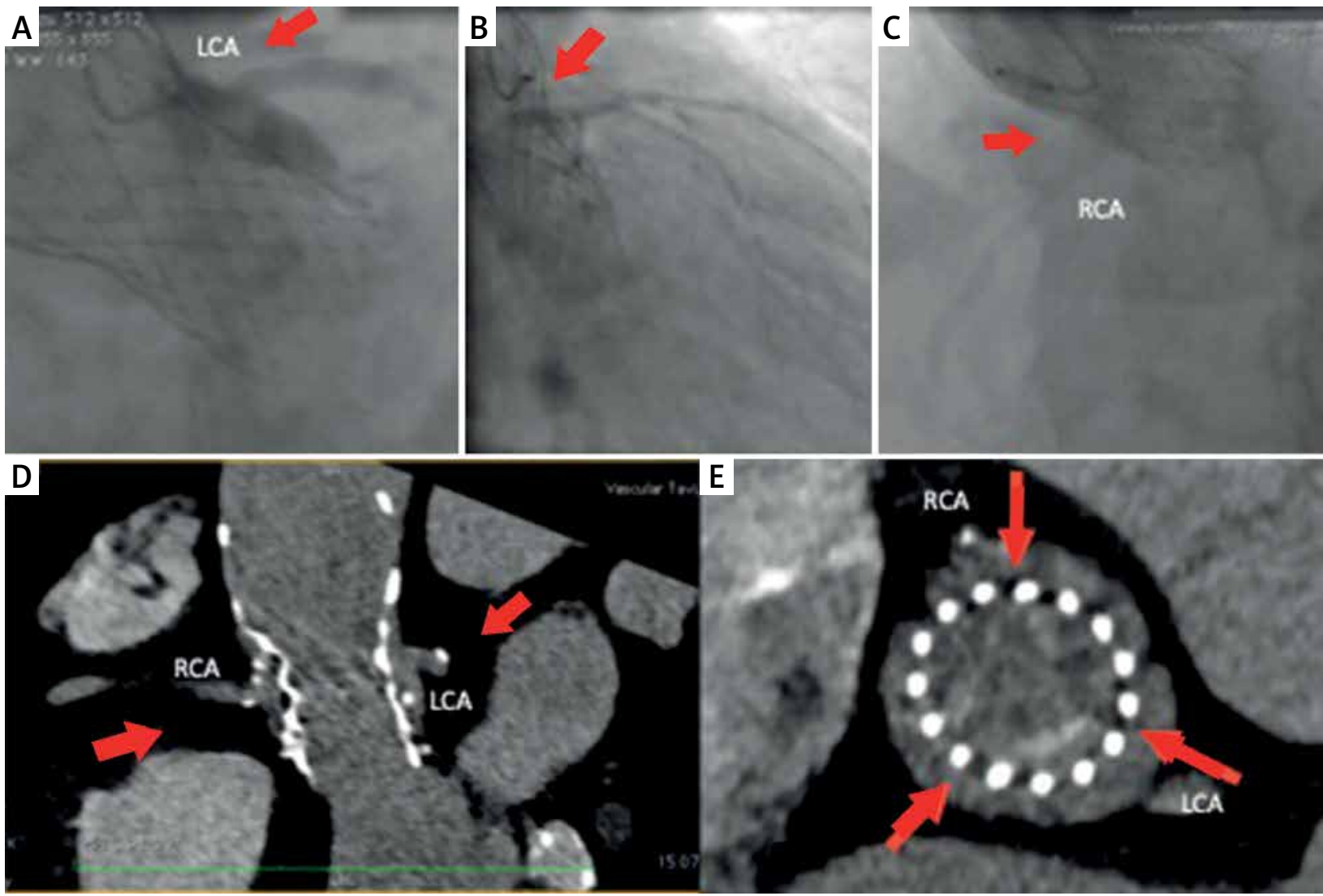

Figure 4. Difficulties in coronary angiography after implantation of the CoreValve 26 prosthesis with unfavorable position of the prosthesis 
comorbidities: a history of ischemic stroke, carotid and lower limb atherosclerosis, diabetes, atrial fibrillation, renal failure, chronic obstructive pulmonary disease (COPD) and pleural effusion. Due to infection and renal failure, the coronary angiography was postponed by a few days to prepare the patient. A Judkins right 3.5 catheter was introduced to the RCA and a Judkins left 4.0 catheter was introduced to the LCA in a relatively easy manner, and the coronary angiography revealed an $80 \%$ LAD stenosis in the proximal segment and $90 \%$ narrowing of the distal OM1 segment (Figures $5 \mathrm{~A}, \mathrm{~B}$, red arrow). No restenosis was found in the previously implanted stents. PCI to LAD was performed using a Judkins left 4.0 guiding catheter and $\mathrm{BMW}$ guidewire with the implantation of the Xience $3.5 \times 15 \mathrm{~mm}$ stent (Figure $5 \mathrm{C}$ red arrow) with a good angiographic result (Figure $5 \mathrm{D}$ red arrow). MSCT before valve implantation shows the coronary artery ostia (Figures $5 \mathrm{E}, \mathrm{F}$ red arrows).

\section{Example VI}

The case of an 86-year-old patient with severe aortic valve stenosis and an Evolut R 26 valve implanted through direct aortic access. The patient died 6 days after the procedure due to multi-organ failure and hemorrhagic complications. The access to the coronary arteries was examined post-mortem and X-ray examination was performed in two perpendicular views.
It was found that the new commissures of the Evolut $R$ valve (Figure 6 C, D black arrows, Figure $6 \mathrm{E}$ white arrows) were located just opposite the coronary ostia (Figures $6 \mathrm{C}-\mathrm{E}$ red arrows). The probe entered the left coronary artery, passed diagonally to the aortic bulb, while the probe which entered the right coronary artery did not pass through the stent struts of the Evolut R prosthesis (Figure $6 \mathrm{C}-\mathrm{E}$ ). As can be seen in the image from post-mortem examination and in the $\mathrm{X}$-ray image, the space between the stent struts and the bulb is minimal (Figures $6 \mathrm{~A}, \mathrm{~B}, \mathrm{~F}$ ). The origin of the right coronary artery is directed upwards (Figures $6 \mathrm{~A}, \mathrm{~B}, \mathrm{~F}$ ). Additionally, the high level of valve implantation could cause the ostium of the right oronary artery to be localized below the outer sealing skirt (Figure $6 \mathrm{~F}$ black two-sided arrow).

For these reasons, we can predict that maneuvering the catheters would be very difficult. Due to the location of commissures of the prosthesis opposite the coronary ostia, oblique direction of the probe from the left coronary artery, and difficult origin of the right coronary artery, the performance of coronary angiography through the stent struts would be very difficult or impossible.

\section{Discussion}

TAVI is a dynamically developing method for the treatment of aortic stenosis. Good results obtained using this method allowed TAVI indications to be expanded to treat
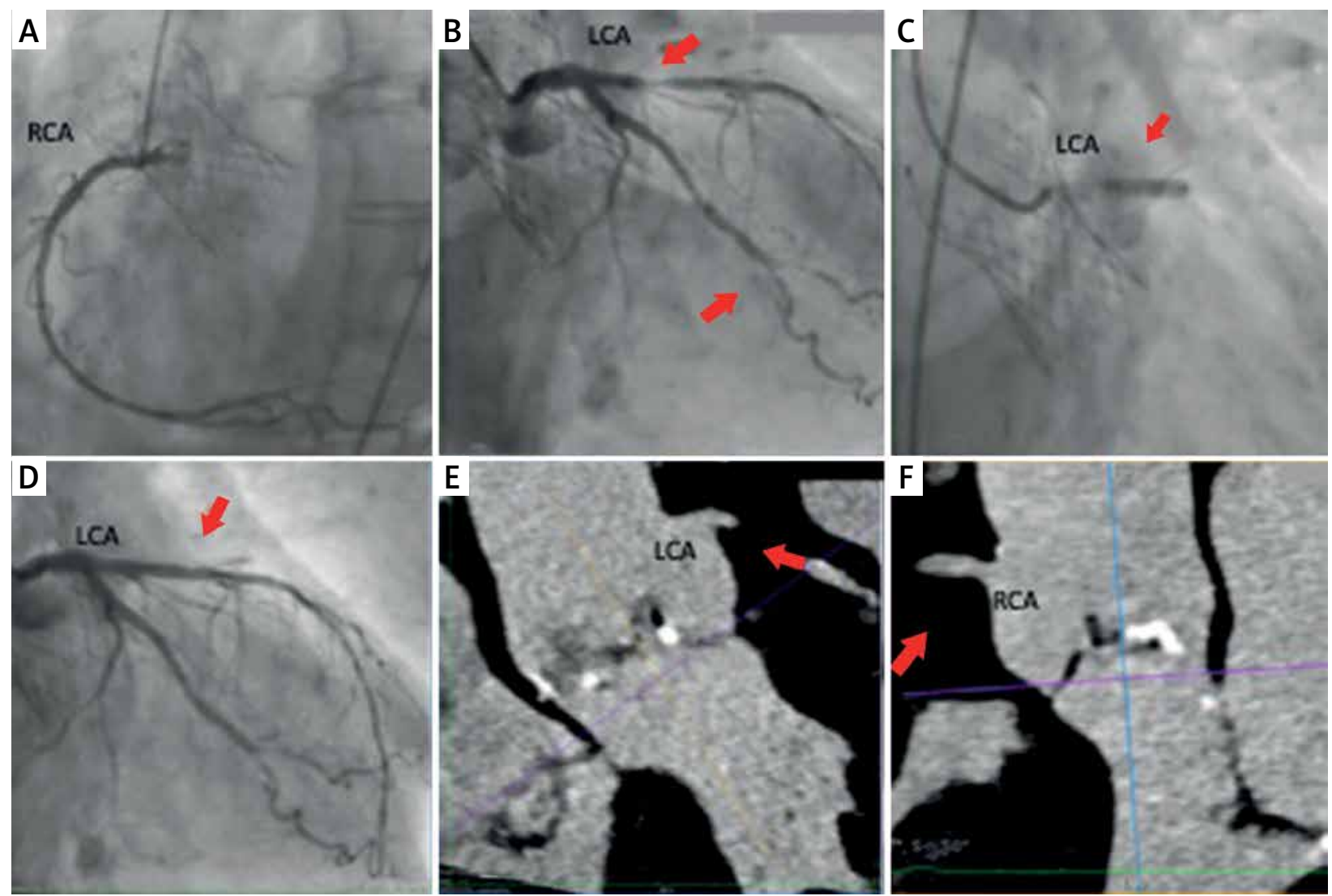

Figure 5. Easy access to the coronary arteries after Evolut R 26 valve implantation 

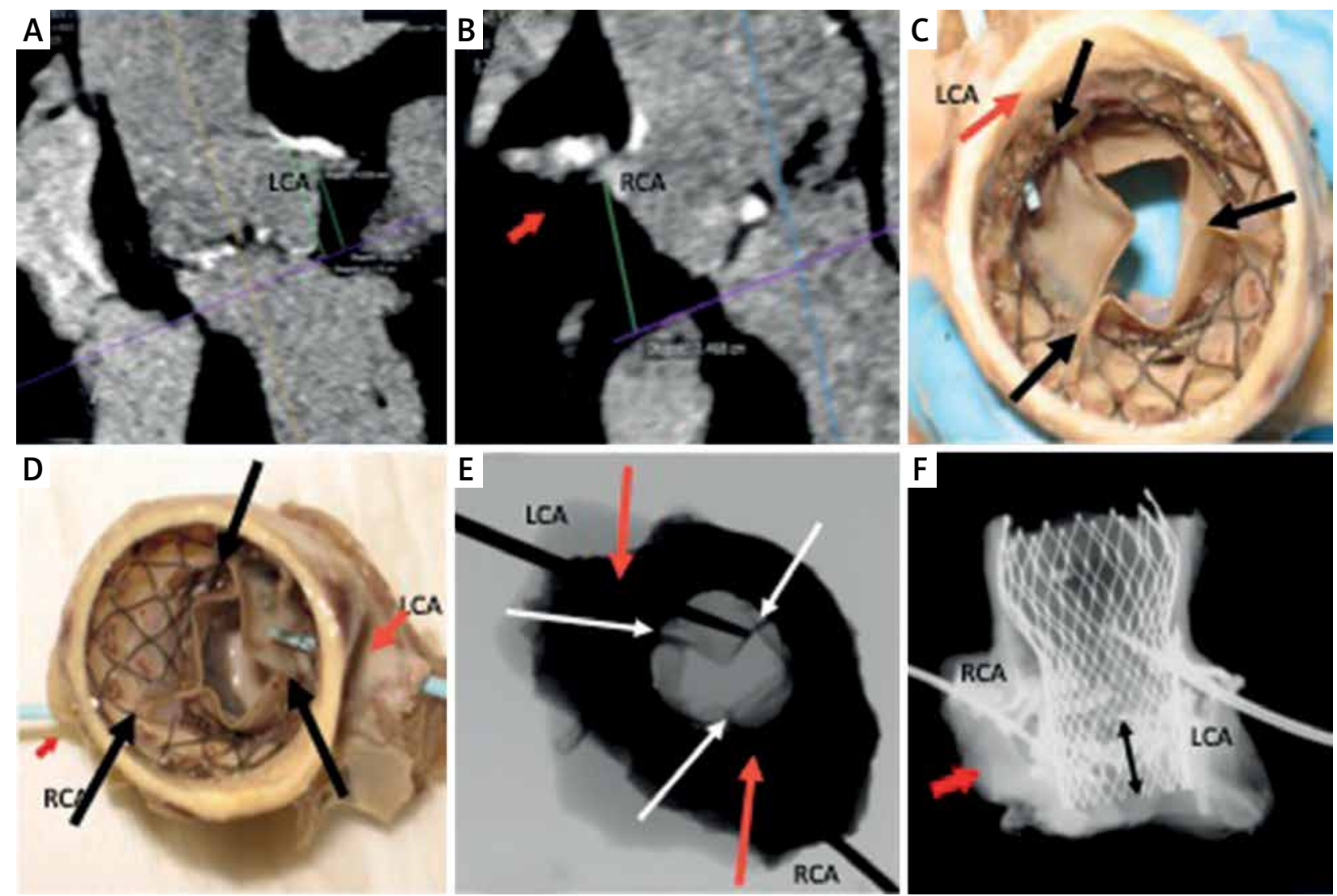

Figure 6. Difficult access to the coronary arteries resulting from their unfavorable anatomy and position of the implanted valve

intermediate-risk patients according to the 2017 guidelines of the ESC [4]. Further spread of TAVI indications in terms of age will depend on the outcome of long-term observations concerning the durability of the aortic valve and, therefore, the possibility of using them in younger patients. A high proportion of patients with coronary artery disease among TAVI patients, as well as similar risk factors for coronary artery disease and aortic stenosis, will probably lead to the situation in which not only aortic valve durability, but also effective invasive treatment of coronary artery disease after TAVI, will be of great significance in terms of survival. There are few reports on the access to coronary arteries after TAVI. Blumenstein et al. reported coronary angiography and $\mathrm{PCI}$ in 35 out of 1000 patients who underwent TAVI [6]. In 15 patients in whom aortic valves were implanted below the coronary ostia (Sapien and JenaValve), coronary angiography could be performed using standard catheters. In the remaining 20 patients in whom aortic valves were implanted and covered coronary ostia partially or completely (CoreValve, Acurate, Portico), selective coronary angiography could not be performed in 9 patients, whereas full access could only be obtained in 3 patients. Boukantar et al. presented problems with coronary angiography related to the presence of prosthesis. They reported that selective coronary angiography after CoreValve implantation was successful in only 9 out of 16 patients [7].

In the study presented by Htun et al., selective angiography of the left coronary artery was possible in $97 \%$ of cases (42/43), and angiography of the right coronary artery was possible in $90 \%$ of cases (29/32). Successful $\mathrm{PCl}$ was performed in 25 patients [8]. Allali et al. evaluated the $\mathrm{PCl}$ procedures after TAVI using the CoreValve. PCI was performed in 17 patients and was successful in 16 of them. It was unsuccessful in one patient with STEMI and the patient died [3]. Zivelonghi et al. performed coronary angiography in 66 patients; in $98 \%$ of cases (65/66) it was selective angiography, and in one coronary angiography was non-diagnostic due to high implantation of the Evolut R valve. In 17 patients PCI was effective [9].

On the other hand, there were no considerable difficulties with access to coronaries in another prospective study. Pesarini et al. conducted a prospective study on the evaluation of the significance of stenoses prior to and after TAVI in patients with severe aortic stenosis and coexisting coronary artery disease [10]. They evaluated the possibility of using the fractional flow reserve (FFR) technique during coronary angiography prior to and after TAVI, as well as the results of such measurements. CoreValve or Evolut $R$ aortic valves were implanted in 14 out of 57 patients, whereas Sapien 3 was used in the remaining 
43 patients. FFR measurements could not be performed in only 1 (1.8\%) patient after TAVI due to difficulties with performing selective coronary angiography. They also argued that it is more beneficial to perform FFR tests after TAVI due to their higher reliability. Therefore, access to coronary arteries after TAVI is of even higher importance.

Post-TAVI access to coronary arteries should be simple and easy. In acute coronary syndromes, it is the intervention time that is of particular significance. Boukantar et al. proved that performing coronary angiography after TAVI procedures is more complicated and related to longer duration of fluoroscopy, higher dose area product and higher use of contrast medium [7]. Procedures after TAVI are related to a higher number of diagnostic catheters, guidewires and guide extension catheters used.

This paper presents examples of both easy and difficult accesses to coronary arteries. Factors that affect the access to coronary arteries are shown in Table I. When the implantation using balloon expandable aortic valves is performed, access to coronary arteries is generally easy. It depends on the implantation at an appropriate level in relation to the aortic annulus and coronary ostia. Our examples of proper implantation showed that it is possible to perform coronary angiography and complex $\mathrm{PCl}$ easily in a patient who received the Edwards Sapien XT/Sapien 3 aortic valve. We also demonstrated that in too high implantation, access to the coronary arteries can be difficult. Katsanos et al. reported that after Edwards Sapien aortic valve implantation, the prosthesis completely covered the orifice of the left coronary artery in $2.2 \%$ of the patients ( 3 out of 142 ), whereas it completely covered the orifice of the right coronary artery in $7.7 \%$ of the patients (11 out of 142). PCl had to be performed in 3 patients, and it was successful in all 3 cases [11].

Balloon expandable aortic valves cannot be repositioned; therefore, all efforts are focused on their positioning during the implantation, as it is in the case of the latest aortic valve delivery system - the Sapien 3 Edwards Commander Delivery System. According to Katsanos et al., too high implantation may be encountered rather frequently, so in $33.6 \%$ of the patients the aortic valve stent covers at least $1 \mathrm{~mm}$ of the orifice of the left coronary artery, whereas it covers at least $1 \mathrm{~mm}$ of the orifice of the right coronary artery in $46.3 \%$ of the patients [11]. Positioning of new commissures in front of the coronary ostia constitutes another important factor. This may be important in cases when the aortic valve is implanted high. This problem was noted by Blumenstein et al. [12]. They developed a method for implanting the Edwards Sapien aortic valve transapically using the DynaCT system, and visualizing commissures of the native and implanted valves so that the commissures overlapped after implantation. Fuchs et al. studied the commissural alignment of the implanted valve in relation to native commissures

Table I. Factors influencing impediment of access to coronary arteries

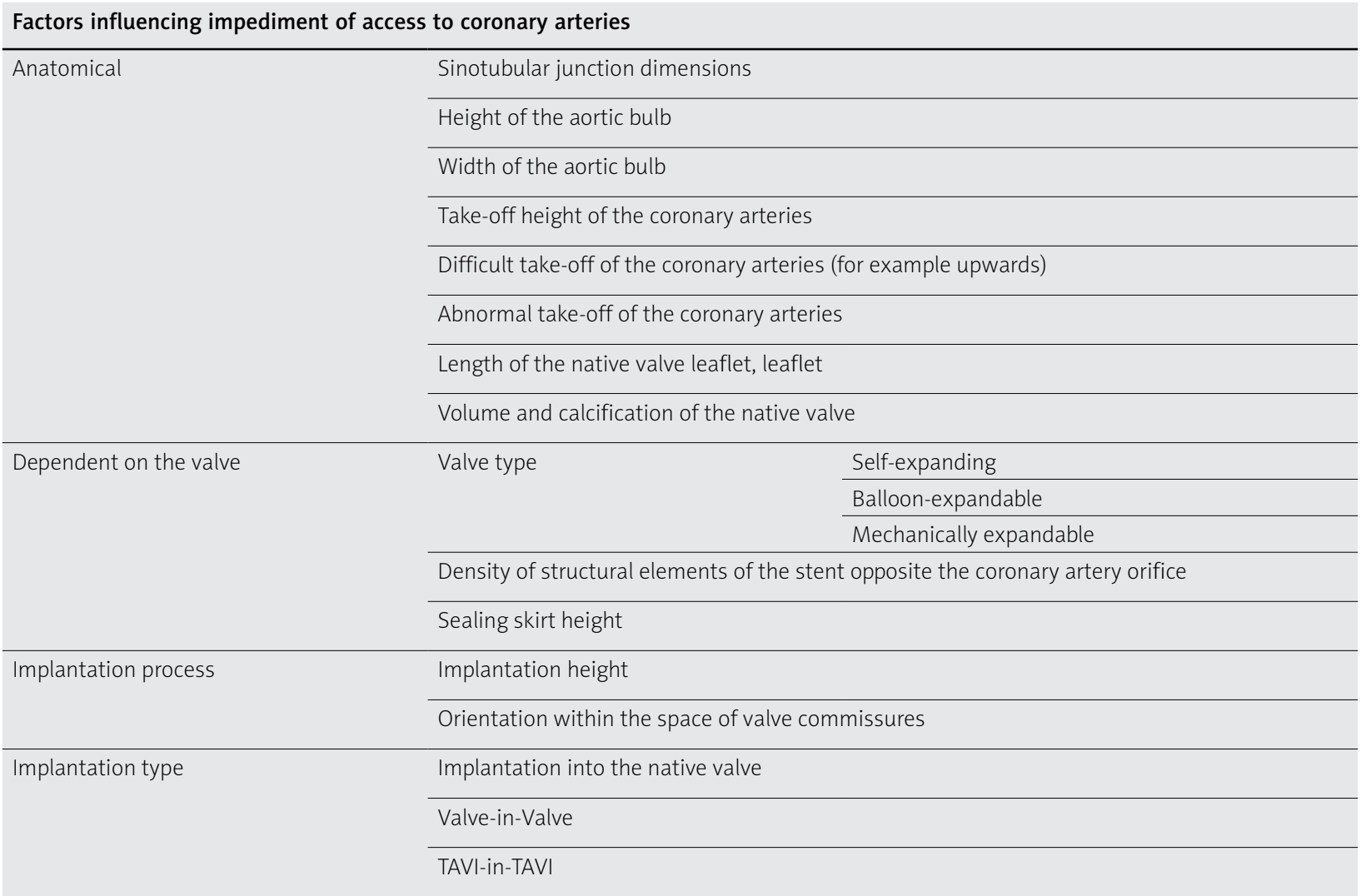


after TAVI and surgical aortic valve replacement (SAVR). In $96 \%(27 / 28)$ of the patients the valve was aligned in relation to the commissures after SAVR, and in the case of the TAVI procedure, as many as $53 \%$ of the patients showed moderate or severe misalignment of the commissures. This means that in more than a half of the patients, regardless of the valve used, conditions impeding access to the coronary arteries were present [13].

In the case of self-expandable aortic valves based on a Nitinol stent, the mesh constituting the stent, which is of various density, depending on the type of aortic valve (CoreValve, Evolut R, Portico), poses some difficulties in positioning catheters or guidewires for coronary angiography, and in some cases, selective insertion into the orifice of a coronary artery is impossible. Another factor that hinders the performance of coronary angiography and $\mathrm{PCl}$ for this type of aortic valve is the position of the new commissures, which may be located in front of the coronary ostia [13]. Due to anatomical differences in the location of the bulb, its displacement, as well as the use of various access routes, so far there has been no reliable method for implanting the aortic valves in such a way that the native aortic valve leaflets were located in front of the implanted aortic valve leaflets and the native aortic valve commissures and implanted aortic valve commissures overlapped. This is of particular importance in the case of a prosthesis with leaflets mounted above the annulus. In the Evolut $\mathrm{R}$ and CoreValve prosthesis, the method for the positioning of a delivery system at an angle of $45^{\circ}$ is imprecise when transfemoral access is used, due to the differences in the orientation of the aortic bulb in individual patients. When it comes to other access routes, the method for the positioning of a delivery system to locate the native aortic valve leaflets and commissures as well as the implanted aortic valve leaflets and commissures in front of each other has not been determined.

Finally, the performance of coronary angiography and $\mathrm{PCl}$ may be hindered by limited aortic root space after a new aortic valve implantation and different locations of coronary ostia. The performance of coronary angiographies and $\mathrm{PCl}$ in patients after the Valve-in-Valve and TAVI-in-TAVI procedures will be an even bigger challenge. The Valve-in-Valve implantation creates a new problem. Before the implantation of the biological prosthesis, the cardiac surgeon cuts out the native valve leaflets and orientates the new valve usually according to the commissures of the native valve. In the event of deterioration of the biological prosthesis, valve implantation using the TAVI method (Valve-in-Valve) may cause the prosthetic valve leaflets to deflect outside and be covered by the stent mesh of the valve implanted by TAVI. The stent mesh and the deflected leaflets of the biological prosthesis will make the access to the coronary arteries difficult. Valve-in-Valve implantation alone poses a threat of sud- den occlusion of the coronary arteries with a frequency of approx. $2.3 \%$ and a high mortality rate of up to $48.6 \%$ [14]. Attempts are made to solve this problem through the percutaneous laceration of the biological prosthesis leaflets during the procedure called BASILICA [15]. The experience in performing coronary angiography after Valve-in-Valve is limited. Even greater problems with access to the coronary artery can be encountered in the case of TAVI-in-TAVI procedures. In these cases, there usually is a double layer of the stent and between them the deflected leaflets of the first valve implanted by TAVI. The first experiments in these cases using the BASILICA method are described.

Our paper deals with some of these aspects. The matter of access to coronary arteries requires further observations and experience. Close attention should be paid to the technique of valve implantation, the implantation level, and the orientation in the space of the leaflets of a newly implanted aortic valve. The development of new aortic valve models may provide easy and reliable access to coronary arteries for coronary angiography and $\mathrm{PCl}$ after TAVI.

\section{Conflict of interest}

Piotr Chodór is a Balton consultant and is an author of the patent application WO2018080328. The other authors declare no conflict of interest.

\section{References}

1. Goel SS, Ige M, Tuzcu EM, et al. Severe aortic stenosis and coronary artery disease-implications for management in the transcatheter aortic valve replacement era: a comprehensive review. J Am Coll Cardiol 2013; 62: 1-10.

2. Duncan A, Ludman P, Banya W, et al. Long-term outcomes after transcatheter aortic valve replacement in high-risk patients with severe aortic stenosis: the U.K. Transcatheter Aortic Valve Implantation Registry. JACC Cardiovasc Interv 2015; 8: 645-53.

3. Allali A, El-Mawardy M, Schwarz B, et al. Incidence, feasibility and outcome of percutaneous coronary intervention after transcatheter aortic valve implantation with a self-expanding prosthesis. Results from a single center experience. Cardiovasc Revasc Med 2016; 17: 391-8.

4. Baumgartner H, Falk V, Bax JJ, et al. ESC Scientific Document Group. 2017 ESC/EACTS Guidelines for the management of valvular heart disease. Eur Heart J 2017; 38: 2739-91.

5. Yudi M, Sharma S, Tang G, et al. Coronary angiography and percutaneous coronary intervention after transcatheter aortic valve replacement. J Am Coll Cardiol 2018; 71: 1360-78.

6. Blumenstein J, Kim WK, Liebetrau C, et al. Challenges of coronary angiography and intervention in patients previously treated by TAVI. Clin Res Cardiol 2015; 104: 632-9.

7. Boukantar M, Gallet R, Mouillet G, et al. Coronary procedures after TAVI with the self-expanding aortic bioprosthesis medtronic CoreValve $^{\mathrm{TM}}$, not an easy matter. J Interv Cardiol 2017; 30: 56-62.

8. Htun WW, Grines C, Schreiber T. Feasibility of coronary angiography and percutaneous coronary intervention after transcatheter aortic valve replacement using a Medtronic ${ }^{\mathrm{TM}}$ self-expand- 
able bioprosthetic valve. Catheter Cardiovasc Interv 2018; 91: 1339-44

9. Zivelonghi C, Pesarini G, Scarsini R, et al. Coronary catheterization and percutaneous interventions after transcatheter aortic valve implantation. Am J Cardiol 2017; 120: 625-31.

10. Pesarini G, Scarsini R, Zivelonghi C, et al. Functional assessment of coronary artery disease in patients undergoing transcatheter aortic valve implantation: influence of pressure overload on the evaluation of lesions severity. Circ Cardiovasc Interv 2016; 9: pii: e004088.

11. Katsanos S, Debonnaire P, van der Kley F, et al. Position of Edwards SAPIEN transcatheter valve in the aortic root in relation with the coronary ostia: implications for percutaneous coronary interventions. Catheter Cardiovasc Interv 2015; 85: 480-7.

12. Blumenstein JM, Van Linden A, Moellmann H, et al. DynaCTguided anatomical rotation of the SAPIEN XT valve during transapical aortic valve implantation: proof of concept. Thorac Cardiovasc Surg 2013; 61: 409-13.

13. Fuchs A, Kofoed KF, Yoon SH, et al. Commissural alignment of bioprosthetic aortic valve and native aortic valve following surgical and transcatheter aortic valve replacement and its impact on valvular function and coronary filling. JACC Cardiovasc Interv 2018; 11: 1733-43.

14. Ribeiro HB, Rodés-Cabau J, Blanke P, et al. Incidence, predictors, and clinical outcomes of coronary obstruction following transcatheter aortic valve replacement for degenerative bioprosthetic surgical valves: insights from the VIVID registry. Eur Heart J 2018; 39: 687-95.

15. Dvir D, Khan J, Kornowski R, et al. Novel strategies in aortic valvein-valve including bioprosthesic valve fracture and BASILICA. Eurolntervention 2018; 14: AB74-82. 